\title{
Analysis of immunological resistance to primary Mycobacterium tuberculosis infection in humans
}

January Weiner III', Teresa Domaszewska ${ }^{1}$, Simon Donkor ${ }^{2}$, Philip C. Hill, ${ }^{2,3}$, Jayne S. Sutherland ${ }^{2}$

1. Max Planck Institute for Infection Biology, Berlin, Germany

2. Vaccines and Immunity Theme, Medical Research Council Unit The Gambia at London School of Hygiene and Tropical Medicine, Banjul, The Gambia

3. Otago University, Otago, New Zealand

\section{Corresponding author:}

Dr. Jayne Sutherland, MRC Unit, PO Box 273, Banjul, The Gambia.

Phone: +220 3170701 .

Email: jsutherland@mrc.gm 


\section{Abstract}

Background: Despite recent advances in diagnosis and treatment, tuberculosis (TB) remains a major infectious disease killer in resource-poor settings. Strategies to prevent Mycobacterium tuberculosis (Mtb) infection are urgently required. By characterising natural protective immunity to Mtb infection we aimed to identify correlates of protection to guide vaccine development and other immune based therapies.

Methods: Two groups of Mtb-exposed contacts of TB patients were recruited in The Gambia and assessed for Mtb infection status using either tuberculin skin test (TST) reactivity at baseline and 3 months or QuantiFERON (QFT) reactivity at baseline and 6 months. For both groups, converters were defined as having a negative test at baseline and a positive one at follow-up, while those with a negative test at both time-points were defined as non-converters (Mtb resisters). Participants were analysed using RNA-sequencing and plasma Mtb proteome $\lg \mathrm{A}$ and $\lg \mathrm{G}$ arrays.

Results: Several genes were found to be differentially expressed at baseline between the groups prior to any signs of infection by current tests. Modular analysis revealed a distinct B cell gene signature in TST non-converters compared to converters (at $q<10^{-6}, A \cup C>0.7$ ), which was only present in the most highly exposed group. Interestingly, when infection status was defined by QFT, enrichment of Type I IFN and antiviral gene signatures was observed. Plasma IgG and IgA antibody reactivity across the entire Mtb proteome showed the best differentiation in individuals with the highest exposure. An AUC of $1.0\left(q<10^{-3}\right)$ was observed for $\lg A$ reactivity to Rv0134 and an AUC of 0.98 for $\lg A$ reactivity to both Rv0629c and Rv2188c (all lower in TST non-converters). IgG reactivity to Rv3223c resulted in an AUC of $0.96\left(q<10^{-4}\right)$ and was again lower in TST nonconverters. The highest AUC for those with lower Mtb exposure were 0.84 (Rv2411c) for $\lg A$ and $0.83(\mathrm{Rv} 2131 \mathrm{c})$ for $\lg \mathrm{G}$.

Conclusions: These data provide insight into the early protective response to Mtb infection and possible avenues for novel therapeutic strategies to prevent Mtb infection. 


\section{Introduction}

Despite recent advances in diagnosis and treatment, tuberculosis (TB) remains is the leading infectious disease killer globally. Over 2 billion people are latently infected with Mycobacterium tuberculosis (Mtb) with 10 million new cases and 1.6 million deaths each year [1]. Strategies to prevent Mtb infection occurring in the first place are a high priority. A recent study showed that sustained QuantiFERON (QFT) conversion, which may best reflect the establishment of Mtb infection, was reduced by $45.4 \%$ with Bacillus Calmette-Guérin (BCG) re-vaccination of adolescents in a high-transmission setting [2]. An understanding of the underlying mechanism(s) for protection against infection are required in order to inform clinical development of new vaccine candidates and host-directed therapies.

The World Health Organization (WHO) strategy sets ambitious targets to 'End TB' through a multi-pronged attack, including a major escalation of research activities to identify novel approaches [3]. Therapeutics to prevent/clear Mtb infection is an alternative strategy that could turn the tide on the TB epidemic. Evidence for natural resistance to Mtb infection is best illustrated by the Lübeck disaster where $\sim 20 \%$ of infants vaccinated with Mtb-contaminated BCG did not get infected [4]. In addition, studies of sailors in long-term confinement with a TB patient showed a surprisingly low level of infection [5]. It has also been shown that a proportion of highly Mtbexposed household contacts never acquire Mtb infection [6]. Importantly, longitudinal studies of such individuals confirm a much lower rate of progression to TB disease than those test positive for LTBI [7] suggesting that they are protected and not merely anergic to the antigens in diagnostic tests.

Several cell subsets have been proposed to control resistance to Mtb infection including macrophages, non-classical $T$ cell subsets and B cells. Macrophage-dependent pathways that prevent bacterial uptake or rapidly clear Mtb before the development of an adaptive immune response define innate resisters while adaptive resisters are individuals in which $\mathrm{T}$ cell and $\mathrm{B}$ cell effector functions eliminate or restrict Mtb infections, either independently of IFN-Y production or through priming by non-protein antigens [8]. Further study is required to determine whether adaptive resisters have greater resistance to progression to active TB than individuals with 
traditionally defined LTBI [8]. Genome-wide association analysis has also identified loci that are associated with innate or adaptive resistance to Mtb infection [9].

The aim of this study was to analyse transcriptomic and antibody signatures in Mtb infection converters and non-converters to identify subsets and pathways for rational development of novel interventions to enhance protection against Mtb infection. 


\section{Methods:}

\section{Study participants}

Household contacts of TB index patients were recruited following written, informed consent by the participant or a parent/guardian if $<18$ years. They were screened to rule out active disease and infection status was determined using either Tuberculin skin test (TST) or QuantiFERON (QFT; Qiagen). TST was performed for participants recruited between 2002-2005 at baseline and 3 months using 2 tuberculin units (TU) of purified protein derivative (PPD) RT23 (SSI, Denmark) with a reading $\geq 10 \mathrm{~mm}$ considered positive. QFT (Qiagen, Germany) was performed for participants recruited between 2016 and 2017 at baseline and 6 months according to the manufacturer instructions. Two participant groups were predefined: TST converters (those who were negative $(0 \mathrm{~mm})$ at baseline and converted to positive $(\geq 10 \mathrm{~mm})$ by 3 months) and TST non-converters (those who remained $0 \mathrm{~mm}$ at both time-points). Similarly, analysis of QFT at baseline and 6 months defined QFT converters and non-converters. Whole blood RNA was stabilised in Paxgene RNA blood tubes and stored at -80 until analysis. Heparinised blood was centrifuged $\left(600_{\operatorname{gmax}}, 10\right.$ mins.), the plasma collected and stored at $-20^{\circ} \mathrm{C}$ until analysis. Differential blood counts were performed at baseline for all participants using a hematology analyser (Cell-Dyn, USA).

\section{RNA-sequencing}

RNA was extracted (Qiagen, Germany) and shipped to the Beijing Genome Institute (Hong Kong) and underwent Globin transcript depletion (GlobinClear, Life Technologies, UK). cDNA libraries were prepared using Illumina mRNA-Seq Sample Prep Kit and RNA-Seq was performed by Expression Analysis Inc., at 20 million 50bp paired-end reads, on Illumina HiSeq-2000 sequencers. Read pairs were aligned to the hg19 human genome using gsnap24 which generated splice junction counts for each sample.

\section{Mtb proteome arrays}

$\lg G$ and $\lg A$ serum antibody reactivity to 4000 Mtb antigens was analysed using a full proteome microarray by Antigen Discovery Inc. (USA) as previously described [10, 11]. Briefly, proteome chips were probed with serum from TST converters and non-converters at baseline and 3 months. 
Slides were first blocked for $30 \mathrm{~min}$ in protein array-blocking buffer before incubation with the primary antibody for $2 \mathrm{~h}$. Antibodies were detected with Cy3-conjugated secondary antibodies (Jackson ImmunoResearch) and scanned in a ScanArray 4000 laser confocal scanner (GSI Lumonics, Billerica, MA). Fluoresence intensities were quantified by using QUANTARRAY software (GSI Lumonics).

\section{Statistics}

\section{Participant demographics:}

Data were analysed a using Mann-Whitney U-test or Kruskal-Wallis test with Graph Pad Prism software v7.0 (Software MacKiev, USA).

\section{RNA-sequencing}

RNA Seq count data were processed using the edgeR package. For each batch, only genes with at least 5 counts per million in at least 3 samples were kept. For batch 1, a subset of samples was generated comprising only samples from HIV- individuals older than 17 years. Differential gene expression was analysed using glmLRT function from the edgeR package, which is a log-likelihood ratio test. Discordance analysis was performed using the disco package. Gene set enrichment analysis was performed using the tmod package. In addition, due to subset differences within each batch the ComBat function from the sva $R$ package was used to remove the effect of sub-batches from logarithmized counts per million transformed data.

\section{Antibody arrays:}

For each antigen, the average intensity of all samples was determined. A Mann-Whitney U-test was performed for analysis between groups and adjusted for false discovery rates using the Benjamini-Hochberg test. The Area under the ROC curve (AUC) was used to determine differential reactivity for each antigen for both $\lg G$ and $\lg A$. 


\section{Results:}

\section{Participant demographics}

For metabolomics and antibody arrays, paired plasma samples from 40 converters and 38 nonconverters at baseline and 3 months were sent to Metabolon and Antigen Discovery for processing respectively. No difference in age or sex was seen between the groups but there was a significantly higher proportion of converters living in the same room as the index case (ie closest proximity; $p=0.034$ ) compared to non-converters (Table 1). For RNA-sequencing, analysis was performed on two batches based on TST (batch 1) or QFT (batch 2). For batch 1, 100 baseline RNA samples were sent to BGI for sequencing. Quality control prior to library preparation showed that only $46 / 100$ samples were of optimal quality for sequencing ( $R I N \geq 7.0)$ presumably due to the long storage duration (up to 13 years). Of these 46 samples, 35 were TST non-converters and 11 were TST converters (Table 1). There was a similar proportion of males in both groups, but a significant difference in age (median[IQR] non-converters = 10[5-17] compared to 20[16-32] for the converters $(p<0.001)$. There was no significant difference in proximity between the groups. For batch 2, samples from 16 QFT non-converters and 11 QFT converters were sequenced (Table 1). No significant difference in age, sex or proximity was seen between the groups. Progression to active disease was assessed in all participants for a 24 month period with only 1 QFT converter progressing after 12 months and none of the non-converters progressing. No progressors were seen in the TST converter/non-converter group.

\section{RNA-sequencing:}

Several differentially expressed genes were observed between converters and non-converters at baseline. For the TST group, the most differentially expressed genes included TMEM56 ( $p=1.25 E-$ 08), RP11-364L4.1 ( $p=4.52 E-08)$, LCN2 $(p=1.81 E-07)$, CLIC2 $(p=3.50 E-07)$ and WASHC3 ( $p=3.54 \mathrm{E}-07$ ) (Table 2). For the QFT group, the most differentially expressed genes included RPS9 ( $p=3.14 E-10)$, MDM4 ( $p=6.09 E-07), \operatorname{HSFX} 3$ (7.6E-07) and IGF1R ( $p=5.25 E-06)$ all of which were upregulated in the converters (Table 3). A full description of differentially expressed genes is shown in supplementary table 1. Due to the significant difference in age between the TST converters and non-converters, we also performed filtered analysis of adults only (Table 4). 
Interestingly in this group the top genes included several coding for immune-regulatory functions including CXCL10 ( $p=3.10 \mathrm{E}-05)$, HLA-DQB1 $(p=8.53 \mathrm{E}-04)$ and CD22 ( $p=1.58 \mathrm{E}-03)$. However, significance was lost after adjusting for FDR, most likely due to low numbers in both groups. CXCL10 (IP-10) showed a significant down-regulation in the non-converters while CD22 (inhibitory receptor for B cell receptor signalling; member of lg superfamily) showed an up-regulation. Interestingly, HLA-DQB1 was highly upregulated in the TST non-converters (logFC = -5.6) suggesting this gene may be involved in protection against Mtb infection. Next we performed gene set enrichment analysis using the Tmod package in R. In the total TST-defined group, there was a strong enrichment in B-cell related genes in the TST non-converters (Fig. 1A), which was still evident in the filtered subgroup (Fig. 1B). TST converters had significant enrichment for TLR8BAFF related genes and monocytes (Fig. 1A) although this was lost when filtering was performed. The responses in the QFT non-converters were dominated by an interferon-Type I/anti-viral gene signature (Fig. 1C). The B cell genes incorporated into M47.0, M47.1 and M69 include BLK, CD19, CD22, CD24, CD72, CD79, CD200, CXCR5, CR2 and FCR1/2. The genes that contribute to the Type I IFN response module (LI.M127) include TAP1, IFIH1, IRF7, PARP9, STAT1, PLSCR1, IFITM1, HERC5, DDX60, USP18, RSAD2 and IFIT1.

It is important to note there were no significant differences in white blood cell counts between converters and non-converters (data not shown). However, due to the differences in proximity (ie exposure) of each participants, we next assessed how this affected gene expression profiles (Fig. 2). Interestingly, in those with the lowest exposure, gene signatures were generally within the innate immune cells with enrichment of neutrophils, monocytes and TLR pathways. The B cell signature was only evident in those with the highest exposure to the index TB case (Fig. 2). This suggests that $B$ cells are involved in the early protective immune response to Mtb infection.

\section{Mtb antibody arrays:}

Analysis of both $\lg G$ and $\lg A$ reactivity to the Mtb proteome showed similar findings with extensive differences in reactivity evident (supplementary Table 2). There was a significant exposure gradient effect with the AUC increasing as proximity to the index case increased (Fig. 3). For example, with IgG reactivity to Rv2131c, Rv0363c and Rv3223c the AUC was $0.73,0.83$ and 0.96 
respectively and with $\lg A$ reactivity to Rv0134 at the closest proximity, the AUC was 1.00 . This indicates that antibody reactivity can be used to discriminate between TST converters and nonconverters. Surprisingly, however, the majority of reactivity was higher in the converters (Fig. 3 (red)) suggesting that the enrichment of B cell genes we saw in the non-converters was not primarily due to enhanced antibody production. However, when the most reactive antigens were analysed, non-converters showed higher responses to Rv0831c (fold increase (fi) 1.93), Rv3038c (fi 2.32), Rv2946c (fi 2.09), Rv3604c (fi 2.53), Rv0726c (fi 1.93) and Rv2396 (fi 2.00) (Fig. 4).

We next analysed reactivity to ESAT-6 specific antigens since this is a dominant secretory antigen from Mtb. Only individuals in the closest proximity to the index case showed any differential antibody reactivity to ESAT-6 antigens with the most significant being $\lg$ G reactivity to Rv3875 $(p=0.0008)$. IgA reactivity to Rv3872 ( $p=0.025)$ and $\operatorname{Rv3871}(p=0.030)$ were both increased in converters compared to non-converters at baseline (supplementary Table 2). 


\section{Discussion:}

We have characterised host transcriptomic and antibody responses to Mtb in TST non-converters and converters prior to any signs of infection as determined by current methods. We have previously shown higher levels of soluble IL-17 in non-converters at baseline [12] suggesting they have encountered Mtb and mounted an immune response and are not simply anergic to antigens in the current tests nor is Mtb blocked by physiological barriers. In this study we saw a distinct B cell gene signature reflected by differential antibody responses in TST converters and nonconverters to multiple Mtb antigens. Interestingly, defining infection based on QFT rather than TST showed a distinct Type I interferon/anti-viral gene signature.

Analysis of RNA from converters and non-converters at baseline highlighted several differentially expressed genes. These included CXCL9, which is a T-cell chemoattractant induced by IFN-y [13]; and BNIP3L (BCL2 Interacting Protein 3 Like), a proapoptotic protein, which may play a role in tumor suppression [14] were both upregulated in converters. Consistent with our findings from metabolomics analysis, we also saw upregulation of SLC14A1, which is involved in urea transportation [15] and LCN2, which is involved in iron sequestration and innate immunity [16]. Both of these genes are important in the anti-microbial response and both were upregulated in converters prior to conversion. Interestingly, when modular analysis was performed, we saw a significant enrichment of B cell genes in non-converters compared to converters, whilst converters had enrichment for monocytes and TLR8-BAFF - important in the early airway epithelial response to Mtb [17]. The B cell genes of interest included CD72, which is thought to mediate B cell-T cell interaction whilst CD19, CD22, CD24 and CD200 are all phenotypic/maturation markers. Interestingly high affinity FCR1/2 genes are enriched in active TB compared to latent TB [18] and was recently shown to be a prognostic marker of progression to active TB in Gambian household contacts [19].

It was interesting to see the distinct gene signatures present when infection was defined by QFT rather than TST. Whilst different mechanisms will be inevitable since TST is based on a delayed type hypersensitivity reaction of $48-72$ hours while QFT is based on overnight in vitro stimulation, the fact these signatures are upregulated in the uninfected groups, suggests they are likely to play a role in resistance. Increased production of Type I interferons (IFNa/ $\beta$ ) as part of the 
anti-viral response inhibits the downstream effects of Type II interferon (IFN- - ) responses known to be critical for Mtb control [20]. Thus it was surprising to see an increase in these genes in the QFT non-converters. However, it has previously been shown that in the absence of a response to IFN- $\mathrm{v}$, type I IFNs play a non-redundant protective role against tuberculosis in mice [21] and suggest type I IFN can limit the number of target cells that Mtb can infect in the lungs while IFN- $y$ enhances their ability to restrict bacterial growth [21]. It has also been shown in experimental models that Type I IFN may play a protective role in the context of BCG-induced immunity and could be targeted to improve preventive vaccination against tuberculosis [22].

The enrichment of $B$ cell genes was unexpected so we decided to analyse $\lg G$ and $\lg A$ plasma antibody reactivity to all 4000 Mtb proteins from these same individuals. TST converters had significantly higher levels of both $\lg G$ and $\lg A$ antibodies to multiple Mtb antigens at baseline, which allowed excellent discrimination between converters and non-converters. This was particularly evident when proximity was accounted for: exposed contacts in closest proximity to the index case who later converted their TST had higher antibody levels at baseline. This suggests that antibodies are playing a role in the response to Mtb infection but are not necessarily protecting the host from becoming infected (although a few antigens elicited significantly higher lgG responses in the non-converters). Since we saw such a distinct B cell signature in the nonconverters, this suggests that other antibody-independent functions of B cells are driving the protection from infection and this is currently being assessed in our laboratory.

In conclusion, despite being clinically healthy and showing no signs of latent infection by current tests, we were able to show differential immune responses in subjects who would later convert to a positive TST compared to those who remained TST negative. Our results support the recent proposed criteria to define resistance by Simmonds et al [8], including the use of both TST and QFT with multiple negative results in the first 12 months following exposure. The interesting B cell and Type I IFN signatures provide an avenue for analysis of mechanisms underlying the protection and could be targeted to enhance resistance to Mtb.

\section{Acknowledgements:}


We would like to thank the National TB control program, participants and their families. We also thank the MRC Gambia TB Clinic staff, TB immunology and TB microbiology laboratory staff. The clinical cohort was funded through the MRC Gambia core funding. All experimental analysis was funded through a TBVAC2020 sub-grant awarded to A/Prof Sutherland (Grant number SEP210138189). 


\section{References:}

1. WHO Global Tuberculosis Report 2017. Available at:

http://www.who.int/tb/publications/global_report/en/

2. Nemes E, Geldenhuys H, Rozot V, Rutkowski KT, Ratangee F, Bilek N, Mabwe S, Makhethe L, Erasmus M, Toefy A, Mulenga H, Hanekom WA, Self SG, Bekker LG, Ryall R, Gurunathan S, DiazGranados CA, Andersen P, Kromann I, Evans T, Ellis RD, Landry B, Hokey DA, Hopkins R, Ginsberg AM, Scriba TJ, Hatherill M; C-040-404 Study Team. Prevention of M. tuberculosis Infection with H4:IC31 Vaccine or BCG Revaccination. N Engl J Med. 2018; 379: 138-149

3. World Health Organization. The End TB Strategy. (2014). Available at: http://www.who.int/tb/post2015_TBstrategy.pdf?ua=1

4. Fox GJ, Orlova M, Schurr E. Tuberculosis in Newborns: The Lessons of the "Lübeck Disaster" (1929-1933). PLoS Pathog. 2016; 12: e1005271k

5. Hawn TR, Day TA, Scriba TJ, Hatherill M, Hanekom WA, Evans TG, Churchyard GJ, Kublin JG, Bekker LG, Self SG. Tuberculosis vaccines and prevention of infection. Microbiol Mol Biol Rev. 2014; 78: 650-71

6. Morrison J, Pai M, Hopewell PC. Tuberculosis and latent tuberculosis infection in close contacts of people with pulmonary tuberculosis in low-income and middle-income countries: a systematic review and meta-analysis. Lancet Infect Dis. 2008; 8: 359-68.

7. Hill PC, Brookes RH, Fox A, Jackson-Sillah D, Jeffries DJ, Lugos MD, Donkor SA, Adetifa IM, de Jong BC, Aiken AM, Adegbola RA, McAdam KP. Longitudinal assessment of an ELISPOT test for Mycobacterium tuberculosis infection. PLoS Med. 2007; 4: e1922

8. Simmons JD, Stein CM, Seshadri C, Campo M, Alter G, Fortune S, Schurr E, Wallis RS, Churchyard G, Mayanja-Kizza H, Boom WH, Hawn TR. Immunological mechanisms of human resistance to persistent Mycobacterium tuberculosis infection. Nat Rev Immunol. 2018; 18: 575-589. Review. 
9. Cobat A, Gallant CJ, Simkin L, Black GF, Stanley K, Hughes J, Doherty TM, Hanekom WA, Eley B, Jaïs JP, Boland-Auge A, van Helden P, Casanova JL, Abel L, Hoal EG, Schurr E, Alcaïs A. Two loci control tuberculin skin test reactivity in an area hyperendemic for tuberculosis. J Exp Med. 2009; 206: 2583-91

10. Davies DH, Liang X, Hernandez JE, Randall A, Hirst S, Mu Y, Romero KM, Nguyen TT, Kalantari-Dehaghi M, Crotty S, Baldi P, Villarreal LP, Felgner PL. Profiling the humoral immune response to infection by using proteome microarrays: high-throughput vaccine and diagnostic antigen discovery. Proc Natl Acad Sci U S A. 2005; 102: 547-52.

11. Kunnath-Velayudhan S, Salamon H, Wang HY, Davidow AL, Molina DM, Huynh VT, Cirillo DM, Michel G, Talbot EA, Perkins MD, Felgner PL, Liang X, Gennaro ML. Dynamic antibody responses to the Mycobacterium tuberculosis proteome. Proc Natl Acad Sci U S A. 2010 Aug 17; 107: 14703-8

12. Coulter F, Parrish A, Declan Manning D, Kampmann B, Mendy J, Garand M, Lewinsohn DL, Riley EM, Sutherland JS. IL-17 production from Th17, MAIT and үס cells in tuberculosis infection and disease. Front Immunol. 2017; 8: 1252

13. CXCL9

14. BNIP3L

15. SLC14A1

16. LCN2

17. TLR8-BAFF

18. FCGR1a in TB

19. TB Risk paper

20. Desvignes L, Wolf AJ, Ernst JD. Dynamic roles of type I and type II interferons in early infection with Mycobacterium tuberculosis. J Immunol. 2012; 188: 6205-6215. 
21. Rivas-Santiago CE, Guerrero GG. IFN-a Boosting of Mycobacterium bovis Bacillus Calmette Güerin-Vaccine Promoted Th1 Type Cellular Response and Protection against M. tuberculosis Infection. Biomed Res Int. 2017; 2017:8796760.

22. Gröschel MI, Sayes F, Shin SJ, Frigui W, Pawlik A, Orgeur M, Canetti R, Honoré N, Simeone R, van der Werf TS, Bitter W, Cho SN, Majlessi L, Brosch R. Recombinant BCG Expressing ESX-1 of Mycobacterium marinum Combines Low Virulence with Cytosolic Immune Signaling and Improved TB Protection. Cell Rep. 2017; 18: 2752-2765 


\section{Figure legends:}

Figure 1: RNA-seq analysis modular enrichment of genes in TST non-converters compared to converters (A, B) or QFT non-converters compared to converters (C). Blue indicates higher expression in non-converters. Effect size (box length) indicates area under the curve (AUC).

Figure 2: RNA-seq analysis showing module enrichment genes in those with low (left) or high

(right) exposure to a TB-index case. B cell enrichment was only seen in those with the highest exposure.

Figure 3: $\lg G$ (top) and IgA (bottom) reactivity to Mtb antigens in TST converters (C) and nonconverters (NC).

Figure 4: Antigens showing the greatest $\lg$ (top) and $\lg A$ (bottom) reactivity in TST converters (C) and non-converters (NC) at baseline. 
Table 1: Participant demographics:

\begin{tabular}{|c|c|c|c|}
\hline & Non-converters & Converters & p-value \\
\hline Plasma & $\mathrm{n}=40$ & $\mathrm{n}=38$ & \\
\hline Age (median[IQR]) & $22[18-34]$ & $26[20-37]$ & ns \\
\hline Males (n(\%)) & $14(35)$ & $14(37)$ & ns \\
\hline \multicolumn{4}{|l|}{ Proximity (n(\%)) } \\
\hline Different house & $15(38)$ & $10(26)$ & ns \\
\hline Different room & $21(53)$ & $17(45)$ & ns \\
\hline Same room & $4(10)$ & $11(29)$ & 0.034 \\
\hline RNA TST & $\mathrm{n}=35$ & $\mathrm{n}=11$ & \\
\hline Age (median[IQR]) & $10[5-17]$ & $20[16-32]$ & $<0.001$ \\
\hline Males $(\mathrm{n}(\%))$ & $18(51)$ & $5(45)$ & ns \\
\hline \multicolumn{4}{|l|}{ Proximity (n(\%)) } \\
\hline Different house & $19(54)$ & $6(55)$ & ns \\
\hline Different room & $14(40)$ & $5(45)$ & ns \\
\hline Same room & $3(9)$ & $2(18)$ & ns \\
\hline RNA QFT & $\mathrm{n}=16$ & $\mathrm{n}=11$ & \\
\hline Age (median[IQR]) & $29[19-47]$ & $18[17-34]$ & ns \\
\hline Males $(\mathrm{n}(\%))$ & $8(50)$ & $4(36)$ & ns \\
\hline \multicolumn{4}{|l|}{ Proximity (n(\%)) } \\
\hline Different house & 0 & 0 & ns \\
\hline Different room & 0 & 0 & ns \\
\hline Same room & $16(100)$ & $11(100)$ & ns \\
\hline
\end{tabular}


Table 2. Differentially expressed genes in the TST group with FDR $\leq 0.01$

\begin{tabular}{|c|c|c|c|c|}
\hline Gene & Log FC & P-Value & FDR & Description \\
\hline TMEM56 & 2.29 & $1.25 \mathrm{E}-08$ & $1.95 \mathrm{E}-04$ & Transmembrane protein 56; protein coding \\
\hline RP11-364L4.1 & 2.83 & $4.52 \mathrm{E}-08$ & 3.54E-04 & Pseudogene \\
\hline LCN2 & 2.42 & 1.81E-07 & 9.43E-04 & Lipocalin 2; iron sequestering; innate immunity \\
\hline CLIC2 & 1.22 & $3.50 \mathrm{E}-07$ & $1.11 \mathrm{E}-03$ & Chloride intracellular channel; regulate cell processes \\
\hline WASHC3 & 0.60 & 3.54E-07 & $1.11 \mathrm{E}-03$ & Endocytosis \\
\hline AC010642.1 & -1.50 & 8.25E-07 & $2.15 \mathrm{E}-03$ & Zinc finger protein pseudogene \\
\hline CCDC153 & 1.43 & $1.25 \mathrm{E}-06$ & 2.79E-03 & Coiled-coil domain containing protein 153 \\
\hline HNMT & 1.28 & $1.60 \mathrm{E}-06$ & 3.01E-03 & Inactivates histamine by $\mathrm{N}$-methylation \\
\hline FAM114A1 & 1.59 & $1.73 \mathrm{E}-06$ & 3.01E-03 & Protein coding gene; neuronal cell development \\
\hline MED20 & 0.92 & 2.03E-06 & $3.18 \mathrm{E}-03$ & Transcriptional co-activator complex \\
\hline RAB10 & 0.80 & 3.17E-06 & 4.36E-03 & Regulate intracellular vesicle trafficking \\
\hline TCF4 & -0.89 & 3.52E-06 & 4.36E-03 & Transcription factor 4; ERK signalling \\
\hline CXCL9 & 2.39 & $3.62 \mathrm{E}-06$ & 4.36E-03 & T cell trafficking; anti-microbial \\
\hline SLC14A1 & 1.80 & 3.95E-06 & 4.42E-03 & Mediates urea transport in erythrocytes \\
\hline ATG3 & 0.67 & 4.56E-06 & 4.76E-03 & Regulation of autophagy during cell death \\
\hline ANAPC13 & 0.79 & 4.93E-06 & $4.82 \mathrm{E}-03$ & Class I MHC antigen processing and presentation \\
\hline TRIQK & 0.75 & 5.89E-06 & $5.20 \mathrm{E}-03$ & Cell growth and maintenance of cell morphology \\
\hline GLRX & 1.10 & $5.98 \mathrm{E}-06$ & $5.20 \mathrm{E}-03$ & Member of glutaredoxin family; antioxidant defense \\
\hline NCOA4 & 0.98 & $8.41 \mathrm{E}-06$ & 6.92E-03 & Androgen receptor binding \\
\hline BNIP3L & 1.26 & $9.52 \mathrm{E}-06$ & 7.45E-03 & Pro-apoptotic subfamily within Bcl-2 family of proteins \\
\hline LINC00662 & 1.23 & 1.05E-05 & 7.68E-03 & Non-coding RNA \\
\hline DPCD & 0.95 & $1.08 \mathrm{E}-05$ & 7.68E-03 & Possible role in the formation/function of ciliated cells \\
\hline ARV1 & 1.09 & 1.15E-05 & 7.84E-03 & ER cholesterol and bile acid homeostasis \\
\hline ARL6IP5 & 0.73 & 1.35E-05 & 8.27E-03 & $\begin{array}{l}\text { Regulates intracellular concentrations of taurine and } \\
\text { glutamate. Expression affected by Vitamin A }\end{array}$ \\
\hline FBXO9 & 0.78 & 1.37E-05 & 8.27E-03 & $\begin{array}{l}\text { F-box protein family; phosphorylation-dependent } \\
\text { ubiquitination }\end{array}$ \\
\hline DENND5B & -1.24 & 1.37E-05 & 8.27E-03 & Promotes the exchange of GDP to GTP \\
\hline TBC1D27 & -1.14 & 1.47E-05 & 8.37E-03 & Pseudogene \\
\hline OLFM4 & 3.14 & $1.50 \mathrm{E}-05$ & 8.37E-03 & $\begin{array}{c}\text { Olfactomedin 4; antiapoptotic factor that promotes } \\
\text { tumor growth }\end{array}$ \\
\hline TMEM55A & 1.02 & 1.57E-05 & 8.49E-03 & Superpathway of inositol phosphate compounds \\
\hline EVPL & 1.42 & $1.81 \mathrm{E}-05$ & $9.44 \mathrm{E}-03$ & $\begin{array}{l}\text { Member of the plakin family of proteins; component of } \\
\text { desmosomes and epidermal cornified envelope }\end{array}$ \\
\hline IGFBP7 & 0.97 & 1.98E-05 & 9.79E-03 & $\begin{array}{c}\text { Member of the insulin-like growth factor (IGF)-binding } \\
\text { protein (IGFBP) family }\end{array}$ \\
\hline DCAF6 & 0.72 & 2.03E-05 & 9.79E-03 & Ligand-dependent coactivator of nuclear receptors \\
\hline FAM26F & 1.32 & 2.07E-05 & 9.79E-03 & Calcium Homeostasis Modulator Family Member 6 \\
\hline
\end{tabular}


Table 3. Differentially expressed genes in the QFT group with FDR $\leq 0.05$

\begin{tabular}{|c|c|c|c|c|}
\hline Gene & Log FC & P-Value & FDR & description \\
\hline RPS9 & 5.39 & $3.14 \mathrm{E}-10$ & 4.87E-06 & ribosomal protein S9 \\
\hline MDM4 & 0.73 & $6.09 \mathrm{E}-07$ & 3.97E-03 & MDM4, p53 regulator \\
\hline HSFX3 & 6.04 & 7.66E-07 & 3.97E-03 & heat shock transcription factor family \\
\hline RP60S & -0.92 & 4.10E-06 & 1.59E-02 & 605 ribosomal protein pseudogene \\
\hline IGF1R & 0.72 & $5.25 \mathrm{E}-06$ & 1.63E-02 & insulin like growth factor 1 receptor \\
\hline STRN3 & 0.80 & $9.23 \mathrm{E}-06$ & $1.80 \mathrm{E}-02$ & striatin 3; calmodulin binding protein \\
\hline RPL39P3 & -0.85 & 9.27E-06 & $1.80 \mathrm{E}-02$ & ribosomal protein L39 pseudogene 3 \\
\hline PCNA & -0.63 & $9.60 \mathrm{E}-06$ & $1.80 \mathrm{E}-02$ & proliferating cell nuclear antigen \\
\hline PCK2 & -0.62 & $1.18 \mathrm{E}-05$ & $1.80 \mathrm{E}-02$ & mitochondrial \\
\hline POLR2L & -0.63 & $1.26 \mathrm{E}-05$ & $1.80 \mathrm{E}-02$ & RNA polymerase II subunit $L$ \\
\hline TFP1 & 5.47 & $1.31 \mathrm{E}-05$ & $1.80 \mathrm{E}-02$ & transferrin pseudogene 1 \\
\hline SYNE3 & 0.59 & 1.39E-05 & 1.80E-02 & Actin binding \\
\hline LAMTOR2 & -0.68 & $1.71 \mathrm{E}-05$ & $1.82 E-02$ & MAPK and MTOR activator 2 \\
\hline RNF181 & -0.60 & 1.73E-05 & 1.82E-02 & ring finger protein 181 \\
\hline NKTR & 0.73 & $1.82 \mathrm{E}-05$ & 1.82E-02 & natural killer cell triggering receptor \\
\hline PMVK & -0.60 & 1.87E-05 & 1.82E-02 & phosphomevalonate kinase \\
\hline C19orf70 & -0.60 & 2.07E-05 & 1.89E-02 & chromosome 19 open reading frame 70 \\
\hline DPM3 & -0.67 & $2.18 \mathrm{E}-05$ & 1.89E-02 & dolichyl-phosphate mannosyltransferase \\
\hline HLA-DMA & -6.98 & $2.51 E-05$ & 2.06E-02 & MHC class II, DM alpha \\
\hline HLA-B & -6.14 & $2.90 \mathrm{E}-05$ & 2.13E-02 & $\begin{array}{c}\text { MHC class I, B } \\
\text { heterogeneous nuclear ribonucleoprotein }\end{array}$ \\
\hline HNRNPH1 & 0.65 & 2.96E-05 & 2.13E-02 & $\mathrm{H} 1$ \\
\hline RARRES3 & -0.67 & 3.02E-05 & 2.13E-02 & retinoic acid receptor responder 3 \\
\hline RBM25 & 0.58 & 4.35E-05 & 2.74E-02 & RNA binding motif protein 25 \\
\hline LILRB3 & -3.44 & 4.46E-05 & 2.74E-02 & leukocyte immunoglobulin like receptor B3 \\
\hline TMSB10 & -0.59 & 4.54E-05 & 2.74E-02 & thymosin beta 10 \\
\hline STX16 & 0.48 & 4.61E-05 & 2.74E-02 & syntaxin 16 \\
\hline FBXO6 & -0.80 & 4.77E-05 & 2.74E-02 & $\begin{array}{c}\text { F-box protein } 6 \\
\text { metastasis associated lung }\end{array}$ \\
\hline \multirow[t]{2}{*}{ MALAT1 } & 0.69 & $5.26 \mathrm{E}-05$ & $2.90 \mathrm{E}-02$ & adenocarcinoma \\
\hline & 0.87 & 5.47E-05 & 2.90E-02 & novel transcript, sense intronic to ST20 \\
\hline RPL29P11 & 2.33 & $5.60 \mathrm{E}-05$ & 2.90E-02 & ribosomal protein L29 pseudogene 11 \\
\hline LMBR1L & 0.47 & $6.41 \mathrm{E}-05$ & 3.20E-02 & limb development membrane protein 1 like \\
\hline TMEM170B & 0.80 & $6.59 \mathrm{E}-05$ & 3.20E-02 & transmembrane protein $170 \mathrm{~B}$ \\
\hline LTB & 5.44 & 7.12E-05 & 3.35E-02 & lymphotoxin beta \\
\hline PLEKHF1 & -0.69 & 7.33E-05 & 3.35E-02 & triggers caspase-independent apoptosis \\
\hline SERPING1 & -1.76 & 7.85E-05 & 3.49E-02 & $\begin{array}{l}\text { complement component } 1 \text { inhibitor } \\
\text { long intergenic non-protein coding RNA }\end{array}$ \\
\hline \multirow[t]{2}{*}{ LINC01089 } & 0.67 & 8.31E-05 & 3.59E-02 & 1089 \\
\hline & 0.96 & $9.11 \mathrm{E}-05$ & 3.83E-02 & uncharacterized LOC100288123 \\
\hline SF3B1 & 0.49 & 9.67E-05 & 3.87E-02 & $\begin{array}{l}\text { splicing factor } 3 b \text { subunit } 1 \\
\text { NDUFA4, mitochondrial complex }\end{array}$ \\
\hline NDUFA4 & -0.50 & $9.71 E-05$ & 3.87E-02 & associated \\
\hline NKG7 & -0.85 & 1.05E-04 & 4.01E-02 & natural killer cell granule protein 7 \\
\hline ULK1 & 0.53 & 1.06E-04 & 4.01E-02 & unc-51 like autophagy activating kinase 1 \\
\hline SRSF11 & 0.57 & 1.15E-04 & 4.14E-02 & serine and arginine rich splicing factor 11 \\
\hline EPSTI1 & -1.18 & 1.17E-04 & 4.14E-02 & epithelial stromal interaction 1 \\
\hline EIF4A1 & 0.98 & 1.17E-04 & 4.14E-02 & eukaryotic translation initiation factor $4 \mathrm{~A} 1$ \\
\hline
\end{tabular}




$\begin{array}{cccc}\text { ATP11A } & 0.47 & 1.23 \mathrm{E}-04 & 4.24 \mathrm{E}-02 \\ \text { PSMG3 } & -0.57 & 1.52 \mathrm{E}-04 & 4.91 \mathrm{E}-02 \\ \text { OAS1 } & -1.46 & 1.53 \mathrm{E}-04 & 4.91 \mathrm{E}-02 \\ \text { SERINC5 } & 0.69 & 1.54 \mathrm{E}-04 & 4.91 \mathrm{E}-02 \\ \text { BANF1 } & -0.57 & 1.58 \mathrm{E}-04 & 4.91 \mathrm{E}-02 \\ \text { FOXK1 } & 0.55 & 1.59 \mathrm{E}-04 & 4.91 \mathrm{E}-02 \\ \text { AQR } & 0.67 & 1.61 \mathrm{E}-04 & 4.91 \mathrm{E}-02 \\ & & & \\ \text { PAN3 } & 0.45 & 1.66 \mathrm{E}-04 & 4.93 \mathrm{E}-02 \\ \text { ECI1 } & -0.55 & 1.68 \mathrm{E}-04 & 4.93 \mathrm{E}-02 \\ \text { ZMYND15 } & 1.10 & 1.73 \mathrm{E}-04 & 4.98 \mathrm{E}-02\end{array}$

ATPase phospholipid transporting $11 \mathrm{~A}$ proteasome assembly chaperone 3 2'-5'-oligoadenylate synthetase 1 serine incorporator 5 barrier to autointegration factor 1 forkhead box K1 aquarius intron-binding spliceosomal factor $\operatorname{poly}(A)$ specific ribonuclease subunit PAN3

enoyl-CoA delta isomerase 1 zinc finger MYND-type containing 15 
Table 4. Differentially expressed genes in the TST group $\geq 15$ years with FDR $\leq 0.05$

$\begin{array}{ccccc}\text { Gene } & \text { Log FC } & \text { p-value } & \text { FDR } & \text { Description } \\ \text { CXCL10 } & 3.97 & 3.10 \mathrm{E}-05 & \text { ns } & \\ \text { MTRNR2L1 } & 4.19 & 3.23 \mathrm{E}-05 & \text { ns } & \text { ns } \\ \text { LILRB2 } & 3.61 & 4.67 \mathrm{E}-04 & \text { ns } & \text { ns } \\ \text { HNMT } & 1.40 & 4.89 \mathrm{E}-04 & 6.91 \mathrm{E}-04 & \mathrm{~ns} \\ \text { EEF1A1P5 } & 1.50 & 7.54 \mathrm{E}-04 & \mathrm{~ns} \\ \text { ADAMTS1 } & 1.51 & 8.53 \mathrm{E}-04 & \mathrm{~ns} \\ \text { HLA-DQB1 } & -5.65 & -82 \mathrm{E}-04 & \mathrm{~ns} \\ \text { SRGAP3 } & -1.10 & 1.12 \mathrm{E}-03 & \mathrm{~ns} \\ \text { HOPX } & 0.85 & 1.58 \mathrm{E}-03 & \mathrm{~ns} \\ \text { CD22 } & -0.84 & 1.65 \mathrm{E}-03 & \mathrm{~ns} \\ \text { CLIC2 } & 1.16 & 1.70 \mathrm{E}-03 & \mathrm{~ns} \\ \text { RP11-394B2.5 } & -0.85 & 1.71 \mathrm{E}-03 & \mathrm{~ns} \\ \text { FAM26F } & 1.60 & 1.74 \mathrm{E}-03 & \mathrm{~ns} \\ \text { CCDC114 } & -1.22 & 1.94 \mathrm{E}-03 & \mathrm{~ns} \\ \text { GSKIP } & 0.67 & 2.08 \mathrm{E}-03 & \mathrm{~ns} \\ \text { MRPS35 } & 0.60 & 2.19 \mathrm{E}-03 & \mathrm{~ns} \\ \text { RP11-374P20.4 } & -1.44 & 2.43 \mathrm{E}-03 & \mathrm{~ns} \\ \text { LCN2 } & 2.07 & 2.68 \mathrm{E}-03 & \mathrm{~ns} \\ \text { AC020951.1 } & -1.17 & 2.74 \mathrm{E}-03 & \end{array}$


TST

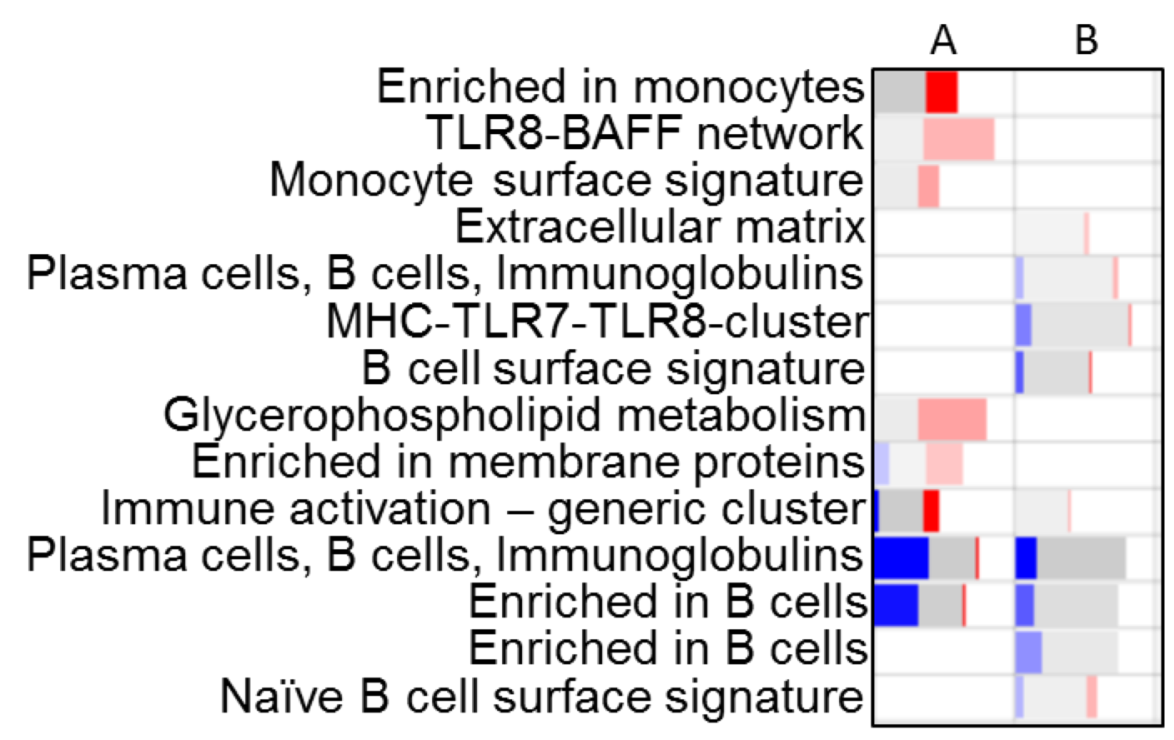

\section{QFT}

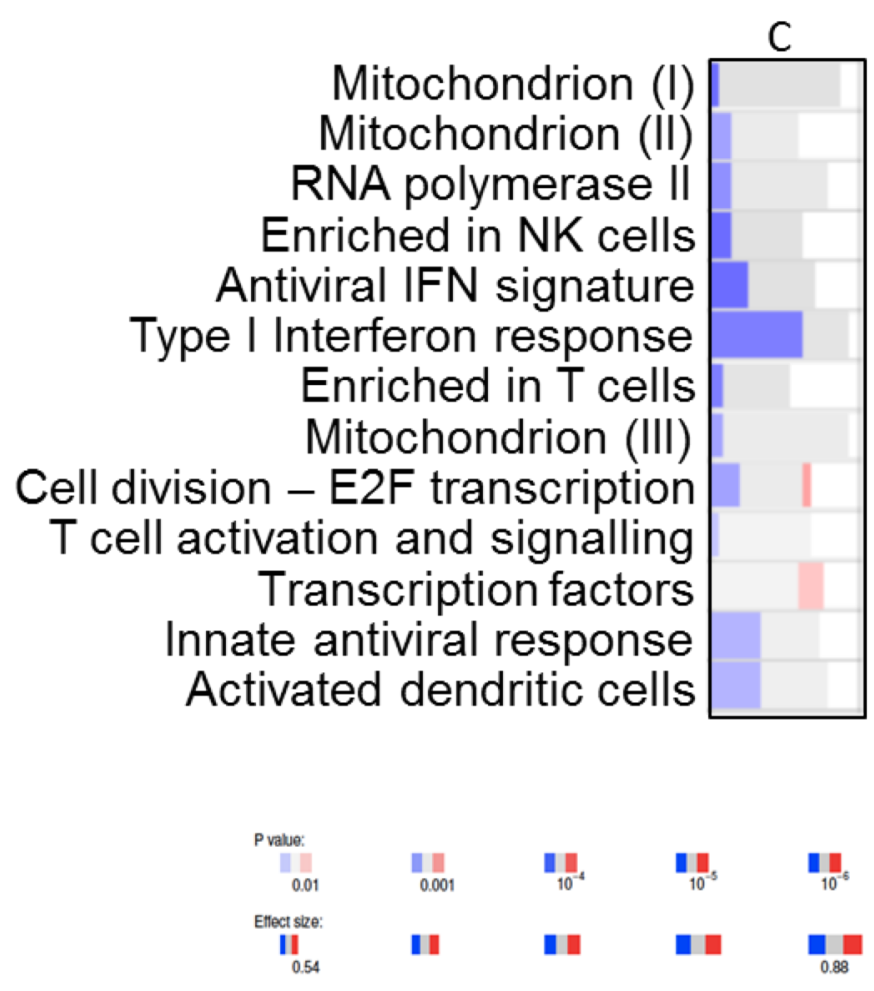




\section{Lowest}

Inflammatory response

Lysosome

Myeloid cell enriched receptors \& transporters

Cell adhesion

Cytoskeleton/actin

Monocyte surface signature

Enriched in monocytes

Lysosomal/endosomal proteins

Enriched in neutrophils

TLR8-BAFF network

Cytoskeletal remodelling

Glycerophospholipid metabolism

Immune activation - generic cluster

Cell cycle and transcription

TLR and inflammatory signalling

Enriched in neutrophils (I)

ATF targets network

Enriched in myeloid cells and monocytes

Extracellular matrix, complement

Enriched in membrane proteins

Enriched in monocytes

Extracellular matrix

\section{Highest}

Enriched in B cells (I)

Enriched in B cells (II)

Mitotic cell cycle in stimulated CD4 T cells

C-MYC transcriptional network

Cell division in stimulated CD4 T cells

Enriched in B cells (III)

Cell cycle (II)

Plasma cells, B cells, Immunoglobulins

$B$ cell surface signature

Plasma cells, Immunoglobulins

Enriched in B cells (VI)

Cell cycle (III)

Mitotic cell cycle - DNA replication

Cell division stimulated CD4+ T cells

E2F transcription factor network

Ran mediated mitosis

Mitotic cycle in stimulated CD4 T cells

MHC-TLR7-TLR8 cluster

PLK1 signalling events

Chromosome $Y$ linked

Mitotic cell division

Cell cycle (I)

Mitotic cell cycle 


\begin{tabular}{cccccc} 
& Antigen & Description & AUC & NC & C \\
\cline { 2 - 6 } & Rv3223c & Alternative RNA polymerase SigH & 0.95 & -0.08 & 0.16 \\
Rv3825c & Polyketide synthase Pks2 & 0.95 & -0.11 & 0.11 \\
Rv2970A & hypothetical protein & 0.93 & -0.01 & 0.25 \\
Rv3299c & Probable arylsulfatase AtsB & 0.93 & -0.14 & 0.02 \\
Rv1469 & Probable cation transporter P-type & 0.91 & -0.17 & 0.10 \\
Rv0101 & Probable peptide synthetase Nrp & 0.91 & -0.07 & 0.10 \\
Rv0006 & DNA gyrase (subunitA) & 0.91 & -0.31 & -0.02 \\
Rv2842c & hypothetical protein & 0.89 & 0.03 & 0.31 \\
Rv1575 & Probable PhiRv1 phage protein & 0.86 & 0.02 & 0.22 \\
Rv1207 & Dihydropteroate synthase 2 FolP2 & 0.86 & -0.11 & 0.19 \\
\hline
\end{tabular}

\begin{tabular}{cccccc}
\multicolumn{1}{c}{ Antigen } & Description & AUC & NC & C \\
\cline { 2 - 6 } IgA & Rv0134 & Possible epoxide hydrolase EphF & 1.00 & -0.36 & 0.04 \\
Rv0629c & Probable exonuclease V (alpha chain) & 0.98 & -0.33 & 0.02 \\
Rv2188c & Mannosyltransferase PimB & 0.98 & -0.39 & -0.04 \\
Rv1041c & Probable is like-2 transposase & 0.95 & 0.04 & 0.45 \\
Rv3507 & PE-PGRS family protein PE_PGRS53 & 0.95 & -0.34 & -0.03 \\
Rv0710 & 30S ribosomal protein S17 RpsQ & 0.95 & -0.15 & 0.25 \\
Rv3596c & Probable ATP-dependent protease & 0.93 & -0.55 & 0.15 \\
Rv1976c & hypothetical protein & 0.93 & -0.24 & 0.16 \\
Rv3666c & Probable periplasmic lipoprotein DppA & 0.93 & 0.63 & 1.50 \\
Rv3905c & Putative ESAT-6 like protein EsxF & 0.93 & -0.44 & -0.14 \\
\hline
\end{tabular}

Red indicates highest reactivity 


\begin{tabular}{|c|ccccc|}
\hline & Antigen & Description & p-value & NC & C \\
\hline Rv0831c & hypothetical protein & 0.0009 & 3.01 & 1.56 \\
Rv3038c & hypothetical protein & 0.0012 & 2.02 & 0.87 \\
Rv2970A & hypothetical protein & 0.0015 & -0.01 & 0.25 \\
\hline Rv2946c & Probable polyketide synthase Pks1 & 0.0025 & 2.44 & 1.17 \\
Rv1575 & Probable PhiRv1 phage protein & 0.0032 & 0.02 & 0.22 \\
Rv3223c & Alternative RNA polymerase SigH (RPOE) & 0.0034 & -0.08 & 0.16 \\
Rv3604c & Probable conserved transmembrane protein & 0.0039 & 4.17 & 1.65 \\
Rv0726c & Possible methyltransferase & 0.0042 & 1.16 & 0.60 \\
Rv3299c & Probable arylsulfatase AtsB & 0.0047 & -0.14 & 0.02 \\
Rv2396 & PE-PGRS family protein PE_PGRS41 & 0.0054 & 3.35 & 1.67 \\
\hline
\end{tabular}

\begin{tabular}{c|ccccc|}
\hline \multicolumn{1}{c}{ Antigen } & Description & p-value & NC & C \\
\hline Rv0134 & Possible epoxide hydrolase EphF & 0.0001 & -0.36 & 0.04 \\
Rv0569 & hypothetical protein & 0.0004 & -0.23 & 0.18 \\
Rv1041c & Probable is like-2 transposase & 0.0005 & 0.04 & 0.45 \\
Rv0629c & Probable exonuclease V (alpha chain) & 0.0005 & -0.33 & 0.02 \\
& Rv3773c & hypothetical protein & 0.0007 & -0.10 & 0.34 \\
Rv3507-s3 & PE-PGRS family protein PE_PGRS53 & 0.0009 & -0.34 & -0.01 \\
Rv0710 & 30S ribosomal protein S17 RpsQ & 0.0011 & -0.15 & 0.25 \\
Rv2188c & Mannosyltransferase PimB & 0.0013 & -0.39 & -0.04 \\
Rv3596c & Probable ATP-dependent protease & 0.0013 & -0.55 & 0.15 \\
Rv2058c & 50S ribosomal protein L28 RpmB2 & 0.0013 & -0.16 & 0.16 \\
\hline
\end{tabular}

